\title{
Dosimetry Assessments in the Irradiation Facilities at the CERN-PS Accelerator
}

\author{
Maurice Glaser, Federico Ravotti, Student Member, IEEE, and Michael Moll
}

\begin{abstract}
At the CERN Proton Synchrotron (PS) accelerator complex, two experimental zones allow the irradiation of samples in a $23 \mathrm{GeV}$ pure proton beam and in a secondary particle environment dominated by $1-\mathrm{MeV}$ neutrons and gamma rays. In this paper, a review of the operative irradiation systems named IRRAD1 and IRRAD2 is presented, as well as the improvements in the techniques used for the beam characterizations and dosimetry.
\end{abstract}

Index Terms-Accelerators, dosimetry, neutron beams, proton beams, radiation monitoring.

\section{INTRODUCTION}

$\mathbf{I}$ $\mathrm{N}$ the experiments of the future CERN Large Hadron Collider (LHC) [1], all materials, optical and electronic components, as well as the particle detectors themselves will be exposed to a harsh radiation environment constituted of electromagnetic and hadronic radiation.

To study radiation damage in semiconductors and other electronic devices [2], since 1998 two irradiation facilities are operated in the East Hall Experimental Area of the Proton-Synchrotron (PS) accelerator at the European Organization for $\mathrm{Nu}-$ clear Research (CERN) [3], [4]. The facility named IRRAD1, located in the PS-T7 beam-line, allows irradiation experiments with the $23 \mathrm{GeV}$ primary proton beam. The IRRAD2 facility, located at the end of the PS-T8 beam line, allows irradiations in a mixed neutron/gamma-ray environment (mainly neutrons of about $1-\mathrm{MeV}$ ) produced in a cavity before a beam stopper.

Over the last few years, the number of irradiation experiments performed annually at these facilities, as well as their complexity, has considerably increased. Experimental assemblies that need on-line readout and experiments performed with non standard beam conditions (high and low intensity, different beam size, special setups for single event effects testing, etc.) are performed more and more frequently. Some examples are given in [5] and [6]. Along with these experiments comes the request for a more precise characterization of the beam profile, the beam intensity and the particle composition of the radiation field. To satisfy these demands, the facilities and the used dosimetry techniques are in a constant evolution. Recently, the facility control system has been fully automated and the radiation levels in the irradiation zones are measured on-line. The radiation level data are stored together with information regarding the object under

Manuscript received September 16, 2005; revised November 13, 2005.

M. Glaser and M. Moll are with the Physics Department, CERN, Geneva CH-1211, Switzerland (e-mail: Maurice.Glaser@cern.ch).

F. Ravotti is with the Technical Support Department, CERN, Geneva CH-1211, Switzerland and also with CEM ${ }^{2}$ University Montpellier II, Montpellier 34095, France (e-mail: Federico.Ravotti@cern.ch).

Digital Object Identifier 10.1109/TNS.2006.880569 irradiation as well as the beam conditions in an on-line database that allows to keep track of all irradiated material [7].

In the year 2004 about 1200 objects have been irradiated. The dosimetry is mainly performed by the activation of Aluminum foils; however techniques like the use of Gafchromic and Optically Stimulated Luminescence based films for beam profiling are extensively employed. Measurements with Silicon radiation detectors, radio-photo-luminescent glasses, polymer-alanine dosimeters and RadFETs for both non-ionizing (NIEL) and ionizing (IEL) energy loss measurements have also been further developed. A part of this development is performed in the framework of the CERN-RADMON Working Group [8] which has the task to provide the LHC machine and the five LHC experiments with radiation monitoring devices.

In this paper we briefly describe the layout and operation of the irradiation facilities (Section II) and then focus on the different techniques used for the characterization of the proton beam (Section III) and the neutron environment (Section IV). Finally, in Section V, the safety issues concerning the irradiated materials at the CERN PS are addressed.

\section{CERN-PS IRRADIATION SYSTEMS}

In the $\mathrm{T} 7$ beam-line the primary $23 \mathrm{GeV}$ proton beam of the PS accelerator is directed to the irradiation area where the facility named IRRAD1, a remote controlled shuttle system, is located. In this zone the proton bursts are delivered during the 16.8s-supercycle of the PS machine in 1-3 spills of about 400 ms each (slow extraction) with a maximal beam intensity of $2 \times 10^{11}$ protons per spill. A defocusing-scanning system is used to spread out the beam in order to produce a uniform irradiation spot over a surface that can vary from 2 to $25 \mathrm{~cm}^{2}$ [3]. Changing these two parameters, proton fluxes from $8 \times 10^{11} \mathrm{~cm}^{-2} \cdot \mathrm{h}^{-1}$ to $8 \times 10^{13} \mathrm{~cm}^{-2} \cdot \mathrm{h}^{-1}$ can be achieved.

The IRRAD1 shuttle moves on a rail inside a metal conduit of a length of about $15 \mathrm{~m}$ and allows transport of the samples to be irradiated directly from the counting room into the irradiation area. The conduit is inserted into a radiation shielding constituted of concrete blocks as shown in Fig. 1. In the irradiation location, to decrease the neutron backscattering to a few percent, a marble absorber of $20 \mathrm{~cm}$ has been inserted between the shuttle position and the following iron beam stopper. A luminescent screen with a camera is used to display the beam spot and a secondary emission chamber (SEC) [9] provides a measurement of the total beam intensity during irradiations.

Fig. 2 shows the layout of the irradiation zone IRRAD2 located at the end of the T8 beam-line. The irradiation is performed in a cavity with secondary particles produced by the primary $23 \mathrm{GeV}$ proton beam after crossing a target constituted of $50 \mathrm{~cm}$ of carbon and $5 \mathrm{~cm}$ of lead [3]. 


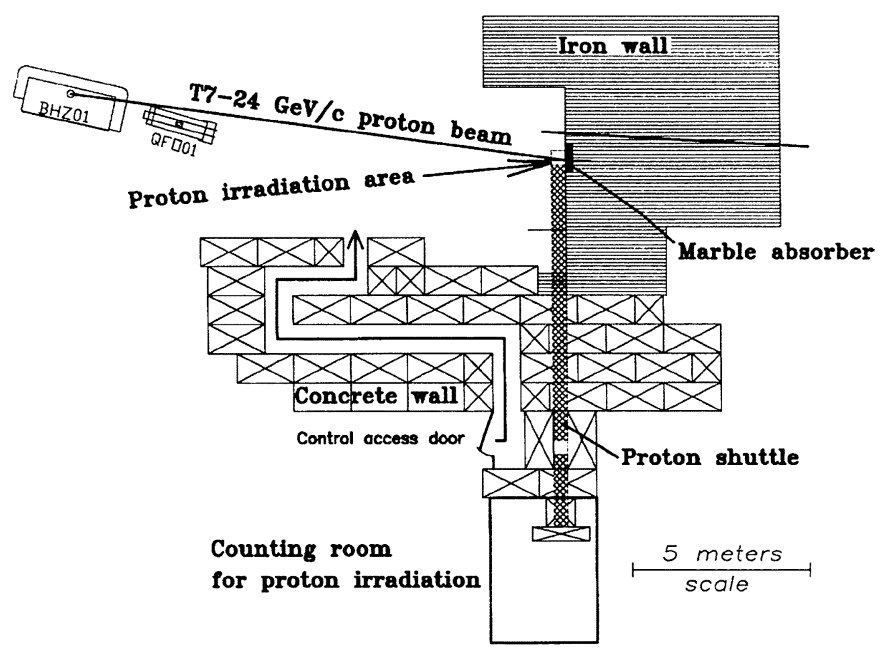

Fig. 1. Layout of the IRRAD1 irradiation facility.

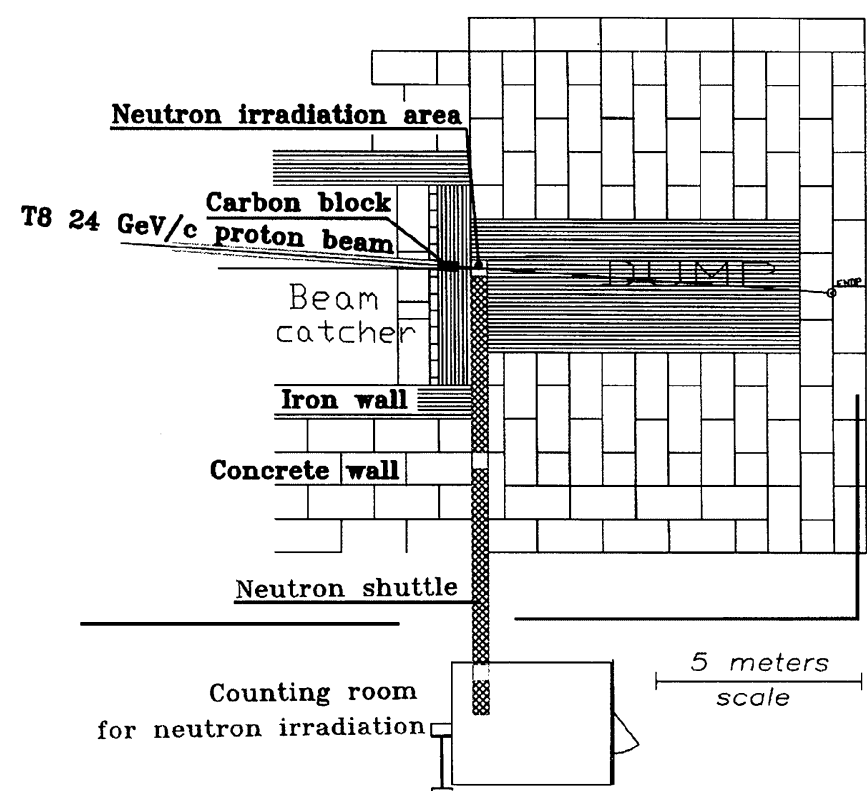

Fig. 2. Layout of the IRRAD2 irradiation Facility.

As for the IRRAD1 facility, a motorized shuttle system allows transport of the samples from the counting room to the irradiation cavity in which a broad spectrum of neutrons, gamma rays and charged hadrons is produced. Fig. 3(a) shows the typical spectrum obtained by Monte Carlo simulations with the FLUKA code [10], while in Fig. 3(b) the three dimensional particle profile inside the irradiation cavity is shown. The secondary particles intensity into the IRRAD2 cavity of Fig. 3(b) has been measured by means of an activation foil technique.

With the remote controlled shuttle system it is possible to set the position of the samples in the vertical coordinate $\mathrm{Z}$ with respect to the beam axis. Depending on the position $Z$, the ratio of charged hadrons in the $\mathrm{GeV}$ energy range to neutrons and gammas can be chosen. It is thus possible to perform irradiations in a pure gamma/neutron environment (at positions far from the beam axis, typically $\mathrm{Z}=50 \mathrm{~cm}$ ) or in a charged-hadron rich radiation environment that better represents the one expected for

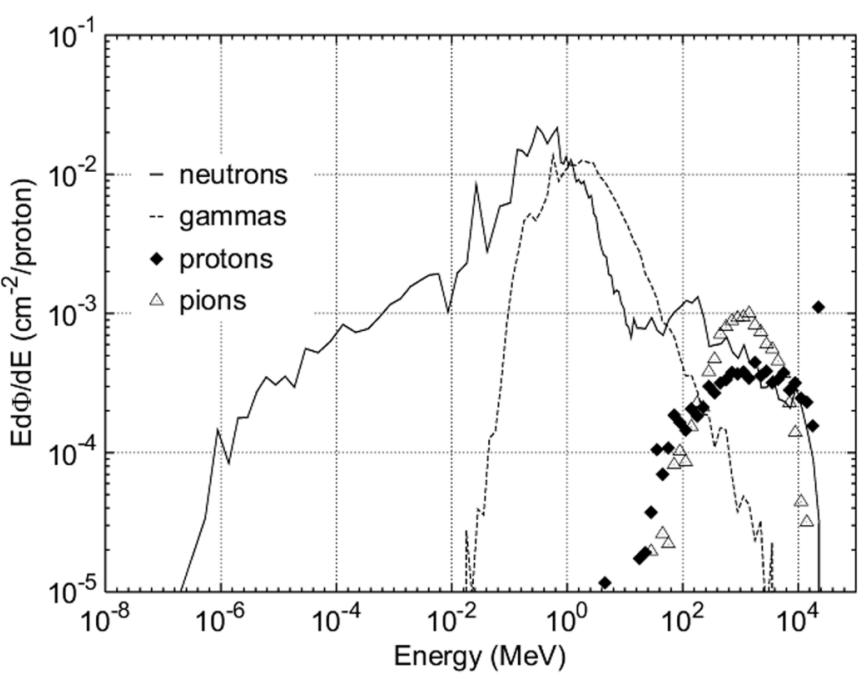

(a)

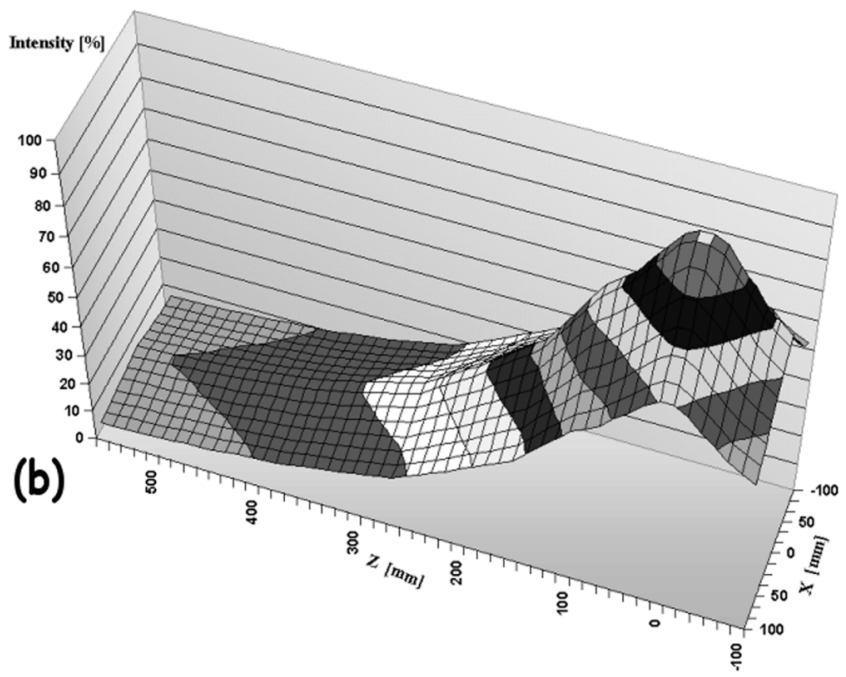

Fig. 3. Particle spectra in IRRAD2 cavity at 10 centimeters from the beam axis normalized to one impinging $23 \mathrm{GeV}$ proton (a) and particle profile as measured by the $\mathrm{Al}$ activation method in the IRRAD2 cavity in function of the distance from the beam axis $\mathrm{Z}(\mathrm{b})$.

the inner detectors of the LHC experiments (at positions close to the beam axis, typically $\mathrm{Z}=10 \mathrm{~cm}$ ).

The proton bursts delivered in the $\mathrm{T} 8$ beam-line have the same time-structure as those of the T7 beam-line. It is therefore possible to reach $1-\mathrm{MeV}$ neutron equivalent fluxes from $0.3 \times 10^{12}$ $\mathrm{cm}^{-2} \cdot \mathrm{h}^{-1}$ to $1 \times 10^{12} \mathrm{~cm}^{-2} \cdot \mathrm{h}^{-1}$ depending both on the number of spills delivered by the PS and the distance of the sample from the beam axis. A SEC [9] provides finally a measurement of the primary proton beam intensity that hits the target.

\section{Proton BeAm CharacteriZATION AND Dosimetry}

\section{A. Activation Measurement of Aluminum Foils}

The basic measurement performed on the $23 \mathrm{GeV}$ proton beam is the determination of the proton fluence by evaluating the ${ }^{24} \mathrm{Na}$ and ${ }^{22} \mathrm{Na}$ activity of Aluminum ( $\mathrm{Al}$ ) foils produced via the nuclear reactions ${ }^{27} \mathrm{Al}(\mathrm{p}, 3 \mathrm{pn}){ }^{24} \mathrm{Na}$ and ${ }^{27} \mathrm{Al}(\mathrm{p}, 3 \mathrm{p} 3 \mathrm{n}){ }^{22} \mathrm{Na}$ respectively [11]. With these activation techniques it is possible to obtain fluence measurements with an accuracy of $\pm 7 \%$. The 
size of the aluminum foils with a thickness of some hundred $\mu \mathrm{m}$ is varying from 0.5 to $2 \mathrm{~cm}^{2}$ according to the size of the samples that have to be irradiated. The half-lives of ${ }^{24} \mathrm{Na}$ and ${ }^{22} \mathrm{Na}$ are about 15 hours and 2.6 years, respectively. According to the time elapsed after irradiation and the irradiation time itself one of the two isotopes is chosen to be measured for the fluence calculation.

Taking into account that ionization is the main contribution to the energy loss of a charged particle and that its mean value, the stopping power $(d E / d x)$, is given by the Bethe-Bloch law, it is possible to simply convert the fluence into the dose (Gy) deposited in thin samples, using the following formula:

$$
D=K \times(d E / d x)_{m} \times \Phi
$$

where $\Phi$ is the proton fluence expressed in particles $/ \mathrm{cm}^{2}, \mathrm{~K}=$ $1.602 \times 10^{-10}$ is a scale factor, and $(d E / d x)_{m}$ expressed in $\mathrm{MeV} * \mathrm{~cm}^{2} / \mathrm{g}$, is the minimum ionizing energy loss rate. For 23 $\mathrm{GeV}$ protons it has values between $1.6-1.8 \mathrm{MeVcm}^{2} / \mathrm{g}$ for materials that are typically irradiated in the facilities. For high-energy charged particles, the contribution of nuclear interactions and the resulting secondaries to the dose in the beam is usually small and can thus be neglected under normal circumstances [12].

Apart from the above described fluence measurements, the beam is also characterized with different types of Gafchromic sensitive medias (Gafchromic is a registered trademark of ISP Corporation) [13] and thin Optically Stimulated Luminescence (OSL) films when a detailed determination of the dose map over the irradiation surface is needed [14].

\section{B. Gafchromic Dosimetric Films}

Gafchromic Dosimetric Films XR, Type-R, HD-810 and MD-55 [13] are currently used for beam profiling, alignment and dose measurements before and during sample irradiation. The films develop a characteristic color upon exposure to ionizing radiation and become progressively darker in proportion to the absorbed dose.

The high sensitivity of the Gafchromic XR Type R film allows an accurate measurement of the proton beam position with a few spills of particles only. The time consumption in the alignment of the IRRAD1 shuttle is thus considerably reduced with respect to the previous procedure which used thin pieces of glass that darkened only after a considerably higher number of proton spills $(\approx 250)$. The films Gafchromic MD-55 and HD-810 are transparent media in which the active layer is coated on thin transparent polyester layers. They develop a characteristic blue color after exposure that can be measured by means of a transmission densitometer. The color density is thus used to measure the absorbed dose during irradiation. Several samples of 1 square centimeter were irradiated in IRRAD1 to obtain the calibration curve shown in Fig. 4 that correlates the color Net Density $\left(\mathrm{N}_{\mathrm{D}}\right)$ with the absorbed dose.

The films were wrapped in a layer of aluminum foil to facilitate handling and irradiated at varying fluences to produce doses from $10 \mathrm{~Gy}$ to more than $1 \mathrm{kGy}$. The optical density of both film types increases with time reaching saturation less than 24 hrs after irradiation. Hence, the readings were performed after

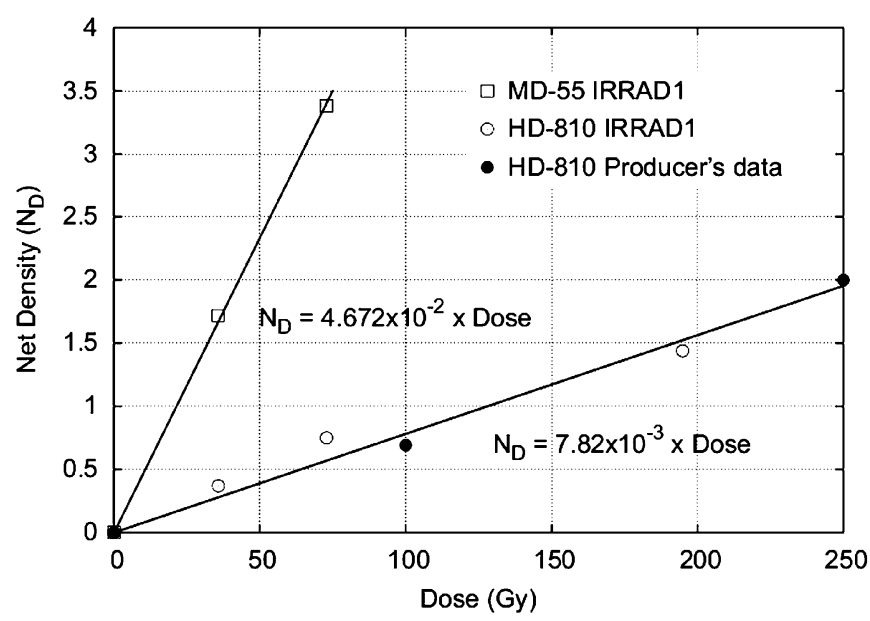

Fig. 4. Gafchromic dosimetric films HD-810 and MD-55. Calibration curves obtained in IRRAD1 facility compared with producer data.

such time for the present calibration [15]. The change in optical density of the films was measured using a Nuclear Associates Model 37-443 densitometer. Unexposed samples were used to provide a base optical density, which was subtracted from the measured one for the $\mathrm{N}_{\mathrm{D}}$ calculation.

\section{Optically Stimulated Luminescent Materials}

The film used for Optically Stimulated Luminescent (OSL) dose mapping is a $100 \mu \mathrm{m}$ thick layer of doped Strontium Sulfide (SrS), silkscreen printed on a kapton foil. After beam exposure, the OSL exhibits a visible emission spectrum when stimulated with near infrared light [16], [17]. The OSL foil reader is a solid-state laser beam (of about $1.0 \mu \mathrm{m}$ wave length) focused on the irradiated foil that is positioned on a X-Y stage [18]. The laser beam is stepped over the foil in the two axes and the emitted luminescent signal (of about $600 \mathrm{~nm}$ wave length) is collected by means of a photo-multiplier tube. Finally the signal is digitized and recorded to get a map of the emitted luminescence versus $\mathrm{X}$ and $\mathrm{Y}$-axis that represents the beam profile. After reading, the material can be completely reset by a 24 hour annealing at $80^{\circ} \mathrm{C}$. The films used at the irradiation facilities were designed to measure doses in the range $0.1-100 \mathrm{~Gy}$.

Their spatial resolution is strongly depending on the dimension of the laser spot used for the readout. With the test bench available in the laboratory of the $\mathrm{CEM}^{2}$ at the University Montpellier II, France it is possible to obtain maps of the proton beam with a $250 \mu \mathrm{m}$ resolution like the examples shown in Fig. 5.

\section{Secondary Electron Emission From Thin Metal Foils}

The need for an on-line method to determine the position and the profile of the PS-T7 proton beam motivated a feasibility study of an instrument based on the proton-induced Secondary Electron Emission (SEE) from thin metal foils [19]. The foils are required to be made of a low cost and relatively short activity lifetime material. Moreover, they have to be on the one hand thin to avoid scattering of beam but on the other hand thick enough to allow easy handling. Aluminum foils were chosen as a good compromise to satisfy most of these requirements. A test setup was realized to verify our calculations and to perform a proof of 

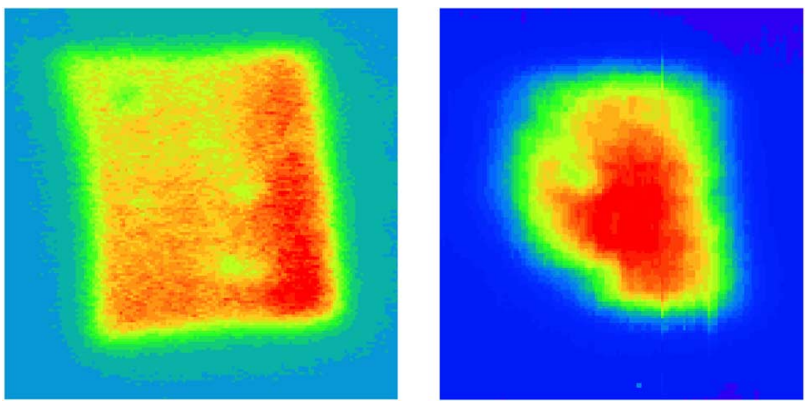

Fig. 5. High-resolution beam profiles measured by a $100 \mu \mathrm{m}$ OSL film. Big beam-spot of about $4.5 \times 4.5 \mathrm{~cm}^{2}$ (left hand side) and standard beam spot of about $2 \times 2 \mathrm{~cm}^{2}$ (right hand side) (Color version available online at http://ieeexplore.ieee.org.).

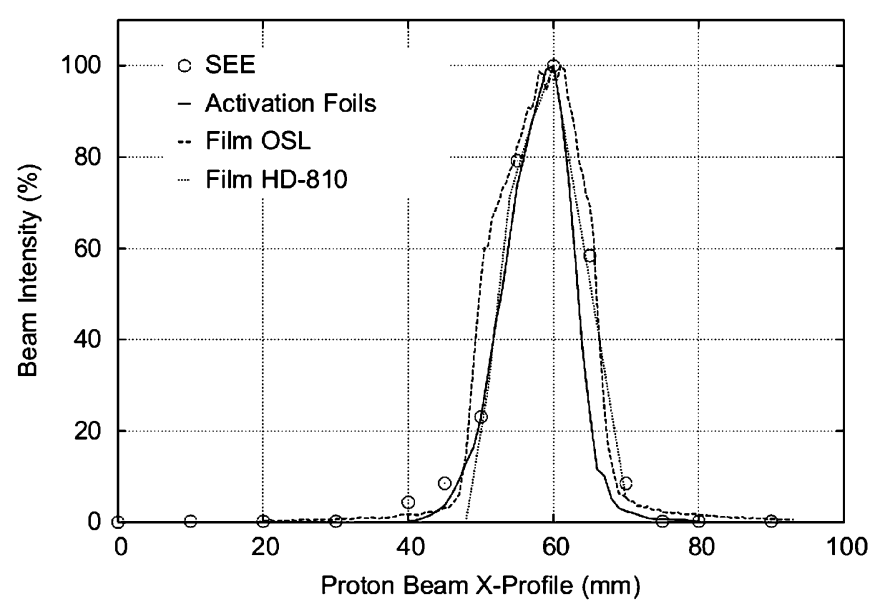

Fig. 6. BPM measurements (circle marks) compared with the profiles obtained by means of OSL, activation foils and Gafchromic films (different continuous lines)

principle before starting the design of a full Beam Profile Monitor (BPM) system.

The BPM test setup consisted of a $3 \times 3 \mathrm{~cm}^{2}, 100 \mu \mathrm{m}$ thick aluminum foil mounted transversally on the IRRAD1 shuttle allowing the beam to pass through the full $3 \mathrm{~cm}$ width of the foil. The foil was electrically connected to a high-gain Transimpedance Amplifier located in the counting room via a 15-meter long coaxial cable. The amplified signal was read out with a LeCroy $9361300 \mathrm{MHz}$ oscilloscope. At a fixed vertical position the foil was displaced to different horizontal positions ranging from 0 to $90 \mathrm{~mm}$ and the charge integrated over one particle spill was measured at each step in order to get a horizontal profile.

Fig. 6 shows the obtained results (circle marks) normalized with respect to the maximum intensity of the beam spot and compared with the same measurement obtained by the profiling techniques mentioned above (various lines). The good agreement of the measurements and the high current collected from the $\mathrm{Al}$ foil (a few $\mathrm{nA}$ in the center of the beam spot) allows us now to proceed with the project by designing a multi-pad detector.

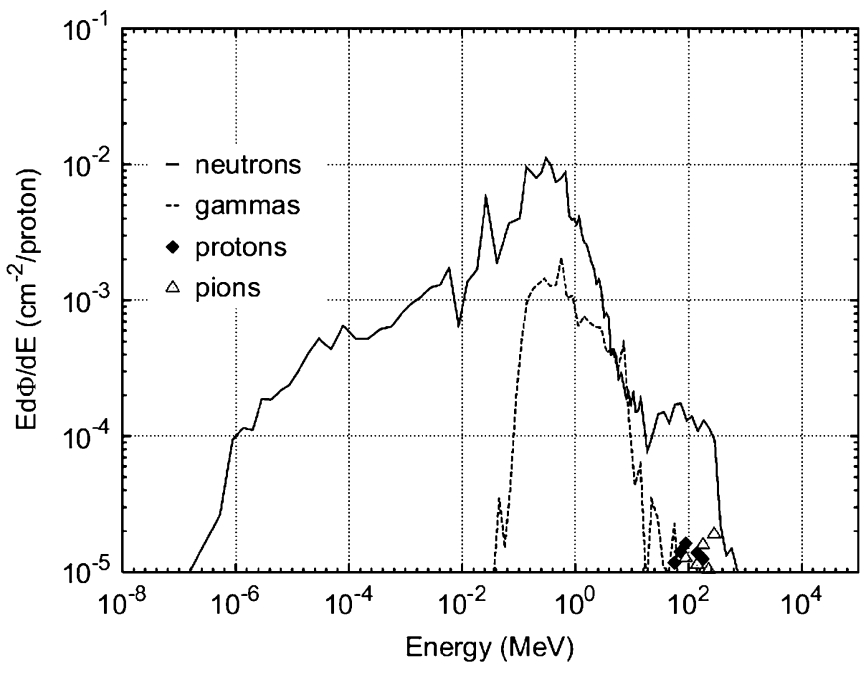

Fig. 7. Particle spectra in IRRAD2 cavity at 50 centimeters from beam axis normalized to one impinging $23 \mathrm{GeV}$ proton.

\section{DOSIMETRY IN THE MiXed Gamma/NeUtron ENVIRONMENT}

Different dosimetry techniques are used to monitor the neutron fluence and the deposited Total Ionizing Dose (TID) in this complex environment depending on the irradiation position and upon the specific user request. For radiation hardness tests and solid-state device characterizations, the environment at $50 \mathrm{~cm}$ distance from the beam axis it is the most used. In this position the radiation field is composed mainly of neutrons $(90 \%)$ with a small gamma ray contamination (10\%). Fig. 7 shows the detail of the particle spectra in this location.

Calculations based on simulations and activation-foil techniques [20] as well as measurements with polymer-alanine dosimeters (PAD), radio-photo-luminescent glasses (RPL) [21], Gafchromic films and silicon detectors [22] were carried out for the calibration of the location at $50 \mathrm{~cm}$ distance from the beam axis. Samples of each dosimeter type were irradiated to ten different neutron fluences.

Monte Carlo simulations with the FLUKA code have been carried out to predict the hadron flux and the production of ${ }^{24} \mathrm{Na}$ in a thin $\mathrm{Al}$ foil placed at $50 \mathrm{~cm}$ from the beam axis. The expected delivered dose in the aluminum, normalized to one proton hitting the target, has also been computed. Measuring the ${ }^{24} \mathrm{Na}$ activity therefore allows calculation of the achieved particle fluence in terms of $1-\mathrm{MeV}$ neutron equivalent fluence $\left(\Phi_{\text {eq }}\right)$.

Fig. 8 shows the production rate of ${ }^{24} \mathrm{Na}$ per incident proton predicted by simulations in $20 \times 20 \mathrm{~cm}^{2} \mathrm{Al}$ foils (continuous line) compared with the measurements carried out with $\mathrm{Al}$ foils placed at different distances from the beam axis ranging from $\mathrm{Z}=-70 \mathrm{~cm}$ to $\mathrm{Z}=+20 \mathrm{~cm}$. The measurements (light dotted lines) taken at steps of $5 \mathrm{~cm}$ with foils of $1 \times 1 \mathrm{~cm}^{2}$, have been normalized to the $20 \times 20 \mathrm{~cm}^{2}$ area (dark dotted line) to allow the comparison with the simulation output. The two sets of ${ }^{24} \mathrm{Na}$ production rates turn out to be in agreement within $20 \%$ for all the irradiation positions (i.e., distances from the beam axis). In 


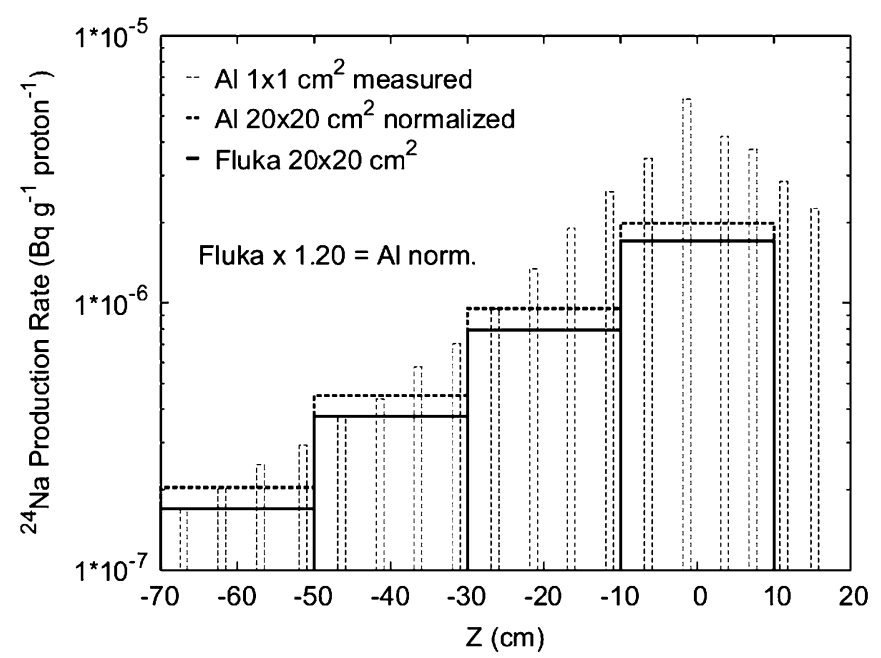

Fig. 8. ${ }^{24} \mathrm{Na}$ Production rate at different distances from the beam axis predicted on $20 \times 20 \mathrm{~cm}^{2} \mathrm{Al}$ foils from Montecarlo simulation (continuous line) compared with the one directly measured with the activation-foils technique (dotted lines).

all of the cases, the measured ${ }^{24} \mathrm{Na}$ production overestimates the corresponding simulated values by about $20 \%$.

Silicon detectors were directly exposed in the IRRAD2 neutron field at ambient temperature without biasing them. After irradiation the devices were annealed for $4 \mathrm{~min}$ at $80^{\circ} \mathrm{C}$ and the increase of the leakage current at full depletion was measured. The leakage current value was then converted to $1-\mathrm{MeV}$ equivalent fluence $\left(\Phi_{\mathrm{eq}}\right)$ following the NIEL scaling in silicon [23]. This method was chosen since it was previously demonstrated that the increase of leakage current per unit of depleted volume for a reverse biased silicon detectors does neither depend on the initial resistivity and conduction type nor on the oxygen and carbon content of the material [22]. They are thus offering a high reproducibility of fluence measurements even if different batches of detectors are used.

The results of this technique to calculate $\Phi_{\text {eq }}$ are shown in Fig. 9. The values obtained by the silicon particle detectors used as NIEL proportional damage counters are here plotted versus the same quantity calculated from Monte Carlo simulations.

With respect to the Monte Carlo simulations the $\Phi_{\text {eq }}$ as measured by the silicon detectors shows a good linearity but is also overestimated by $21 \%$. The reason for these small differences between measurements and the simulation might be manifold. There wasn't possible, after each irradiation step, to measure the activity of the short-lifetime ${ }^{24} \mathrm{Na}$ nuclide and apply it to normalize the simulation output. For this reason, all the simulated values have been scaled using only a few activation measurements performed during the first irradiation steps. Furthermore, although great care was taken to have stable beam conditions during the one week running of the experiment, small variations can not be excluded. And finally the simulation itself and the NIEL scaling of the Silicon detector response might also be afflicted by a small error. Nevertheless, Silicon detectors were identified as a good tool for measuring $\Phi_{\mathrm{eq}}$ and were thus used to plot the experimental data shown hereafter in Fig. 10.

Fig. 10 shows the comparison between the total ionizing dose in aluminum estimated by simulations (empty round markers)

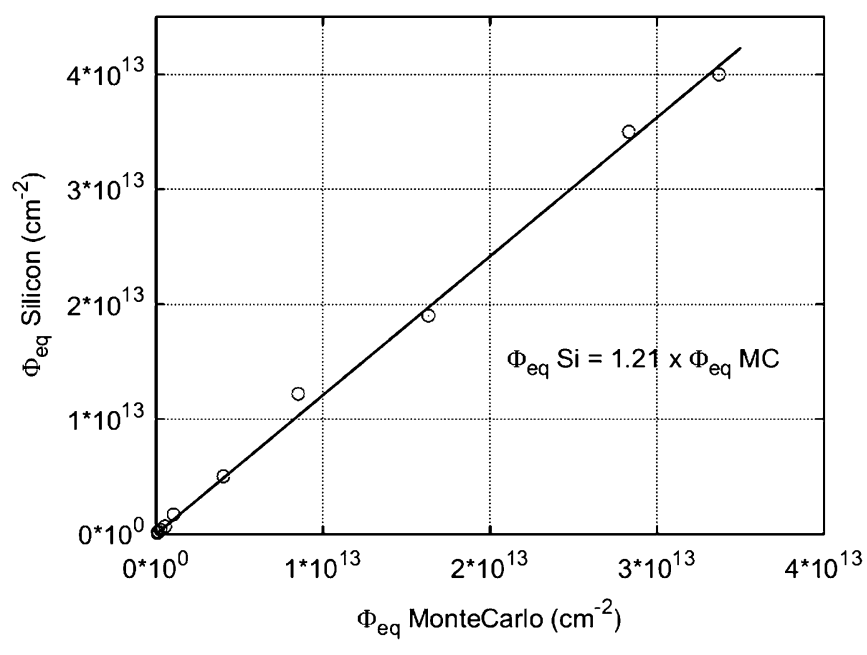

Fig. 9. Equivalent fluence from Silicon detectors, plotted versus the equivalent fluence calculated from Monte Carlo simulations.

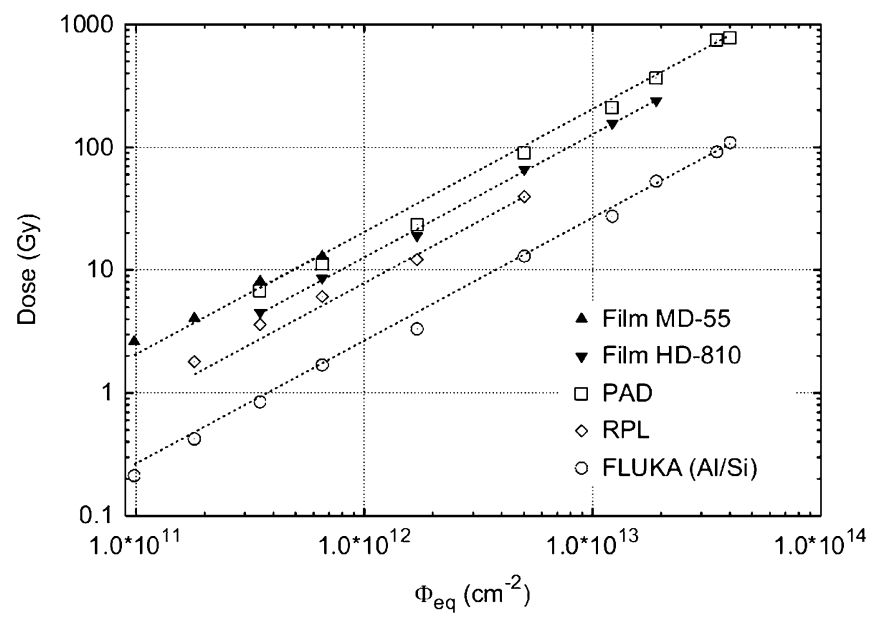

Fig. 10. Comparison among the different TID measured at $50 \mathrm{~cm}$ from the beam axis obtained by different dosimetric means. The dotted lines have been plotted as guidelines within different data sets.

and the values measured with the various dosimeter types (other markers in the plot). The TID deposited by the neutron/gamma environment in thin Aluminum foils is supposed to be equal to the one expected in thin solid-state semiconductors devices.

The values measured with sensitive films, and PAD dosimeters are on average greater by a factor of $7.3 \pm 2.7 \%$ than the value predicted from simulations. The RPL technique shows instead a factor $3.2 \pm 8.5 \%$ greater than simulations. This difference is expected and is due to the different neutron interactions in the various sensitive materials constituting the employed dosimeters [24]. In the IRRAD2 facility the neutron spectrum is dominated by fast and high-energy neutrons (85\% of the neutrons have energies from $10 \mathrm{keV}$ to $1 \mathrm{MeV}$ ). In this range, neutrons deposit a significant fraction of their energy in non-ionizing energy losses and in inelastic interactions that produce fast-light particles that escape from semiconductor materials. The neutron contribution to the TID in semiconductors with respect to the gamma rays is thus in the order of a few tens percent and results in a TID estimation smaller than the one obtained by other systems. This fact has been also proved 


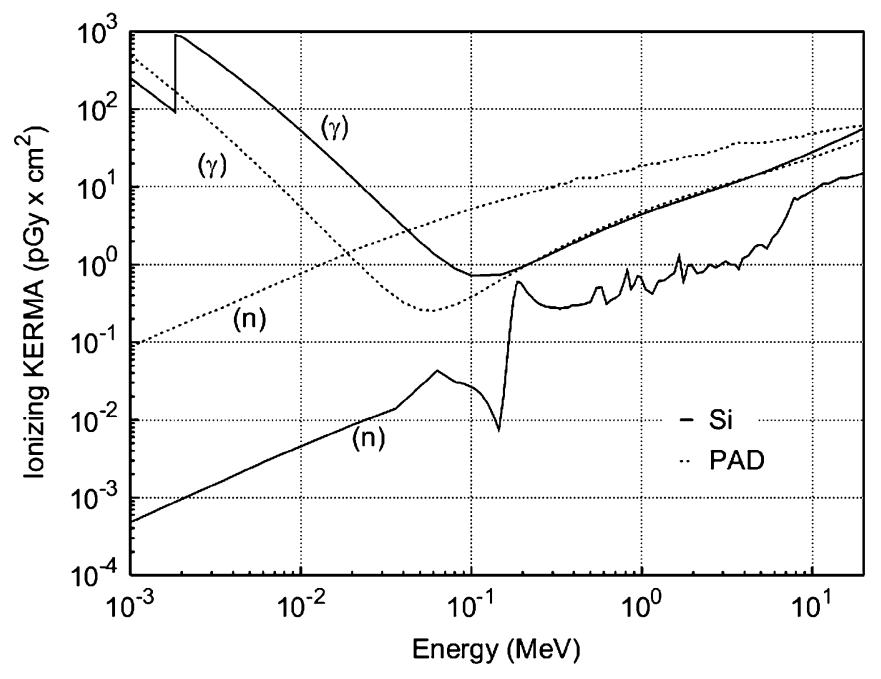

Fig. 11. Neutron (n) and photons $(\gamma)$ KERMA values in Si and PAD in the energy range from $1-\mathrm{keV}$ to $20-\mathrm{MeV}$.

TABLE I

AbSORBED Doses CALCULATED From the PARTICle SPECTRA

\begin{tabular}{|ccc|}
\hline \hline & Silicon & PAD \\
& Gy/proton & Gy/proton \\
\hline Gammas & $\mathrm{D}_{\mathrm{Si}, \gamma}=6.04 \times 10^{-15}$ & $\mathrm{D}_{\mathrm{PAD}, \gamma}=6.10 \times 10^{-15}$ \\
Neutrons & $\mathrm{D}_{\mathrm{S}, \mathrm{n}}=2.74 \times 10^{-15}$ & $\mathrm{D}_{\mathrm{PAD}, \mathrm{n}}=1.52 \times 10^{-13}$ \\
\hline \hline
\end{tabular}

in measuring doses in IRRAD2 by means of solid-state silicon dosimeters [25].

The most important mechanism responsible for transfer of energy from neutrons to polymeric substances like PAD and Gafchromic films is instead elastic scattering. The recoil proton generated in this way will move through the material depositing the initial neutron energy via ionization leading to the increased response.

The films and PAD measurement are therefore in good agreement and lie in an interval of $\pm 15 \%$. It has to be noted that the film MD-55 provides almost the same measurement of the PAD with an error of about $\pm 5 \%$.

The RPL response is a factor 2 lower with respect to the PAD in the IRRAD2 environment and lies thus in between the Alanine measurements and the simulation results. This discrepancy, already observed in a previous work [21], may be explained with an intermediate neutron interaction mechanism in the different material of the RPL (silver-activated aluminophosphate glass).

For the PAD dosimeters only, the above experimental data were compared to the theoretical calculation of the scaling factors for the TID released in PAD and silicon that is the base material for solid-state dosimeters.

Fig. 11 shows the neutron and photon Ionizing KERMA values simulated for Silicon and PAD calculated from [26] and [27]. Folding these coefficients with the neutron and gamma spectra simulated for the IRRAD2 facility of Fig. 7 enables calculation of the absorbed dose expressed in Gy normalized to one incident proton. The results of these calculations are reported in Table I.
The ratio between the dose as measured in PAD and the dose deposed in Silicon as calculated by Monte Carlo is thus given by the following equation:

$$
\frac{D_{\mathrm{PAD}, \mathrm{IRRAD} 2} \text { (reading) }}{\left.D_{\mathrm{Si}, \text { IRRAD2 }} \text { (calculated }\right)}=\frac{D_{\mathrm{PAD}, \gamma}+k \times D_{\mathrm{PAD}, n}}{D_{\mathrm{Si}, \gamma}+D_{\mathrm{Si}, n}}
$$

where the doses $D$ are the ones reported in Table I, and the coefficient $k$ is the relative sensitivity of Alanine for neutrons compared to gamma rays [28].

The relative response in Alanine to monoenergetic neutrons ranges from 0.36 to 0.7 compared with the response to ${ }^{60} \mathrm{Co}$ photons in the energy range from 0.5 to $15 \mathrm{MeV}$ [29]. On the basis of the neutron spectrum of Fig. 7, a coefficient $k=0.4$ has been found for the IRRAD2 facility.

By substituting all of the above data in (2), the dose ratio PAD versus Silicon in IRRAD2 turns out to be equal to 7.6. This is in good agreement with the experimental measurement presented in Fig. 10 which gave a factor of 7.3.

\section{IRRADIATED SAMPLES HANDLING}

Neutron and proton irradiations induce activation in the exposed materials. Equivalent dose-rates of hundreds of $\mu \mathrm{Sv} / \mathrm{h}$ can be reached at $10 \mathrm{~cm}$ distance from the samples and are essentially due to medium-life (years time scale) gamma and beta emitters generated by nuclear reactions in the materials themselves. Even if the remote controlled systems of the facilities minimize the radiation exposure during sample positioning, the storage and the post-exposure handling have to be well controlled and CERN radiation safety rules applied. These rules are continuously updated by the CERN Safety Commission [30] following the recommendation of the CERN member states in matter of protection against ionizing radiations.

During the samples cool-down period the Irradiation facilities have access to several storage facilities at room and low temperature (e.g., $-30^{\circ} \mathrm{C}$ ), equipped to manipulate samples under safe conditions.

After appropriate cool-down (that depends on both irradiation level and specimen material composition), samples belonging to outside institutes can leave CERN following a new shipping procedure according to the Radiation Protection Procedure n. 13 (RPR-13) of January 2004. Details about these new rules can be found in [30] and [31].

\section{ACKNOWLEDGMENT}

The authors wish to thank Dr. L. Dusseau from Montpellier University, France, and Dr. D. F. Lewis from ISP Advanced Materials Groups, Wayne, NJ, for providing the OSL and the Gafchromic films, respectively, used for beam profiling. We would like to express our thanks also to M. Huhtinen from CERN for the Monte Carlo simulations and the useful discussion about particle interaction in Silicon. Finally, we wish also to thank all operators of the CERN-PS machine, especially R. Steerenberg.

\section{REFERENCES}

[1] The Large Hadron Collider Project Webpage, [Online]. Available: http://www.cern.ch/lhc-new-homepage/. 
[2] A. G. Holmes-Siedle and L. Adams, Handbook of Radiation Effects, 2nd ed. Oxford, U.K.: Oxford University Press, 2002.

[3] M. Glaser, L. Durieu, F. Lemeilleur, M. Tavlet, C. Leroy, and P. Roy, "New irradiation zones at the CERN-PS," Nucl. Inst. and Meth., vol. A426, pp. 72-77, 1999.

[4] M. Glaser, M. Huhtinen, F. Lemeilleur, C. Leroy, P. Roy, and M. Tavlet, "Radiation test facilities in the new PS east hall at CERN," in Proc. 5th Eur. Conf. Radiation and Its Effects Components and Systems, Fontevraud, France, 2000, pp. 136-141.

[5] L. Fernandez-Hernando, "A beam condition monitor for the experimental areas of the LHC," in Proc. 9th European Particle Accelerator Conference (EPAC 2004), Lucerne, Switzerland, Jul. 5-9, 2004.

[6] K. E. Arms, "ATLAS pixel opto-electronics," Nucl. Inst. Meth., vol. A554, pp. 458-468, 2005.

[7] CERN-PH Department Irradiation Facilities Webpage, [Online]. Available: http://cern.ch/irradiation/.

[8] LHC Experiment Radiation Monitoring Working Group, RADMON, [Online]. Available: http://www.cern.ch/lhc-expt-radmon/.

[9] K. Bernier, G. d. Rijk, G. Ferioli, E. Hatziangeli, A. Marchionni, V. Palladino, G. R. Stevenson, T. Tabarelli, and E. Tsesmelis, "Calibration of secondary emission monitors of absolute proton beam intensity in the CERN SPS north area," CERN Yellow Rep. 97-07, 1997.

[10] A. Fassò, A. Ferrari, J. Ranft, and P. R. Sala, "FLUKA: Status and prospective for hadronic applications," Proc. MonteCarlo 2000 Conf., Lisbon, Portugal. Berlin, Germany, Oct. 23-26, 2000-2001, Springer-Verlag, pp. 955-960.

[11] E. Leon-Florian, C. Leroy, and C. Furetta, "Particle fluence measurements by activation technique for radiation damage studies," CERN Divisional Report, CERN-ECP-95-15, 1995.

[12] A. H. Sullivan, A Guide to Radiation and Radioactivity Levels Near High-Energy Particle Accelerators. London, U.K.: Nuclear Technology, 1992.

[13] Gafchromic ${ }^{\circledR}$ Radiochromic Dosimetric Films Datasheets, [Online]. Available: http://www.ispcorp.com.

[14] L. Dusseau, G. Polge, S. Matias, J. R. Vaillé, R. Germanicus, R. Broadhead, B. Camanzi, M. Glaser, F. Saigne, J. Fesquet, and J. Gasiot, "High-energy particle irradiation of optically stimulated luminescent films at CERN," IEEE Trans. Nucl. Sci., vol. 48, no. 6, pt. 1, pp. 2056-2066, Dec. 2001.

[15] N. V. Klassen, "Gafchromic MD-55: Investigated as a precision dosimeter," Med. Phys., vol. 24, no. 12, pp. 1924-1934, 1997.

[16] O. Missous, F. Loup, J. Fesquet, H. Prevost, and J. Gasiot, "Optically stimulated luminescence of rare-earth doped phosphors," Eur. J. Solid State Inorg. Chem., vol. 28/s, pp. 163-166, 1991.

[17] D. Plattard, G. Ranchoux, L. Dusseau, G. Polge, J.-R. Vaillé, J. Gasiot, J. Fesquet, R. Ecoffet, and N. Iborra-Brassart, "Characterization of an integrated sensor using optically stimulate luminescence for in-flight dosimetry," IEEE Trans. Nucl. Sci., vol. 49, no. 3, pt. 3, pp. 1322-1326, Jun. 2002
[18] G. Polge, L. Dusseau, S. Matias, D. Gensanne, D. Plattard, J. Fesquet, and J. Gasiot, "Applications to radiotherapy of a dose-mapping system based on the optically stimulated luminescence," in Proc. IEEE Medical Imaging Conf., Lyon, France, Oct. 2-6, 2000.

[19] V. Pugatch, K. T. Knopfle, and Y. Vassiliyev, "Beam profile imaging target," Nucl. Phys. A 701, pp. 204c-208c, 2002.

[20] H. Vincke, I. Brunner, and M. Huhtinen, Production of Radioactive Isotopes in $\mathrm{Al}, \mathrm{Fe}, \mathrm{Cu}$-Samples by Stray Radiation Fields at Proton Accelerator CERN, CERN-TIS-2002-007-RP, 2002.

[21] F. Coninckx, H. Schonbacher, M. Tavlet, G. Paic, and D. Razem, "Comparison of high-dose dosimetry systems for radiation damage studies in collider detectors and accelerators," Nucl. Inst. and Meth., vol. B83, pp. 181-188, 1993.

[22] M. Moll, E. Fretwurst, and G. Lindström, "Leakage current of hadron irradiated silicon detectors-Material dependence," Nucl. Inst. and Meth., vol. A426, pp. 87-93, 1999.

[23] M. Moll, E. Fretwurst, M. Kuhnke, and G. Lindström, "Relation between microscopic defects and macroscopic changes in silicon detector properties after hadron irradiation," Nucl. Inst. and Meth., vol. B186, pp. 100-110, 2002.

[24] M. H. Van de Voorde, Effects of Radiation on Materials and Components-Megarad Dosimetry CERN, 1969, Yellow Rep. 69-12.

[25] F. Ravotti, M. Glaser, M. Moll, K. Idri, J.-R. Vaillé, H. Prevost, and L. Dusseau, "Conception of an integrated sensor for the radiation monitoring of the CMS experiment at the large hadron collider," IEEE Trans. Nucl. Sci., vol. 51, no. 6, pt. 2, pp. 3642-3648, Dec. 2004.

[26] M. B. Chadwick and H. H. Barschall, "A consistent set of neutron kerma coefficients from thermal to $150 \mathrm{MeV}$ for biologically important materials," Med. Phys., vol. 26, pp. 974-991, 1999.

[27] J. H. Hubbell, "Photon mass attenuation and energy absorption coefficients from $1 \mathrm{keV}$ to $20 \mathrm{MeV}$," Int. J. Appl. Radiat. Isot., vol. 33, pp. 1269-1290, 1982.

[28] Y. Katsumura, Y. Tabata, T. Seguchi, N. Morishita, and T. Kojima, "Fast neutron irradiation effects-III. Sensitivity of alanine systems for fast neutron having an energy of $\sim 1 \mathrm{MeV}$," Radiat. Phys. Chem., vol. 28 , no. 4 , pp. 337-341, 1986.

[29] H. Schraube, E. Weitzenegger, A. Wieser, and D. F. Regulla, "Fast neutron response of alanine probes," Appl. Radiat. Isot., vol. 40, pp. 941-944, 1989.

[30] Documents of the CERN Safety Commission-Radiation Protection (RP) Group, [Online]. Available: http://cern.ch/radiation-protection/

[31] CERN SC-RP Radioactive Shipping Informations, [Online]. Available: http://cern.ch/rp-shipping. 